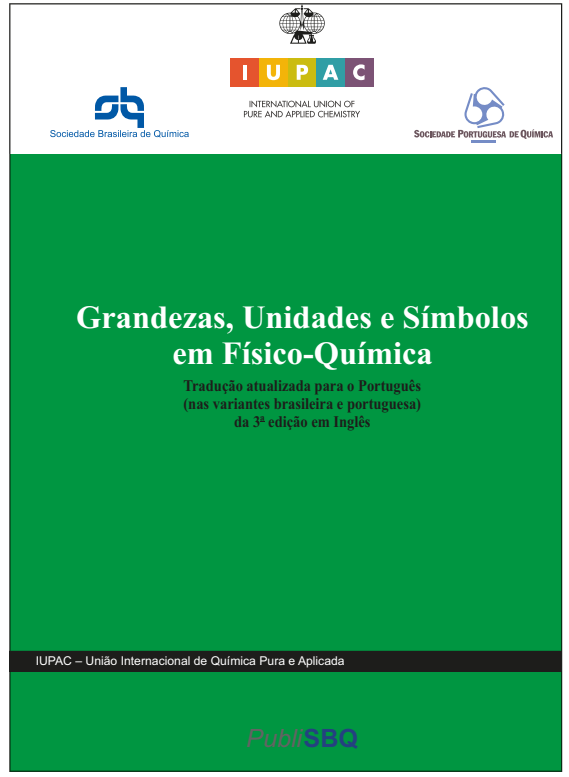

and symbols, is crucial to the success of international science and technology exchanges, with important consequences even for the modern industrial economy. Now, the Livro Verde provides a more homogeneous physicochemical nomenclature in Portuguese, agreed between the chemists of Brazil and Portugal, and with the joint seal of the $S B Q$ and $S P Q$.

In a rapidly expanding scientific literature, where each discipline tends to use its specific language, the Green Book seeks to provide a compilation of widely used terms and symbols drawn from many sources along with simple brief definitions of understanding and explanations of best practice. It is a compilation of the names, symbols and units recommended for physicochemical quantities and includes tables of fundamental constants and conversion factors. In an e-book format, with 272 pages, and with the prospect of an interactive edition in the future, the Livro Verde is the first release of EditSBQ (SBQ's book publishing division) in 2018. The printed version of the Livro Verde will be available in time for the 2018 SBQ Annual Meeting (21-24 May).

Considering that the original English version was published in 2007, this translation updated the values of most of the physical constants (and their conversion factors) as well as some properties of particles, elements and nuclides.

"This book translation is an interesting project because I witnessed for decades the conceptual confusion caused by sloppy terminologies," says Professor Fernando Galembeck (Unicamp), one of the catalysts of this work. "The Green Book guides teachers and students in the use of an organized and uniform language, which facilitates the understanding and use of concepts, as well as contacts, collaborations and interactions in research and professional life."

The Livro Verde in e-book (PDF) format is freely available for download at http://www.sbq.org.br/ livroverde/

\section{Arene Chemistry: Reaction Mechanisms and Methods for Aromatic Compounds}

Jacques Mortier (Ed), Wiley 2016,

ISBN: 978-1-118-75201-2

(http://doi.org/10.1002/9781118754887)

reviewed by Oleg M. Demchuk

The carbocyclic aromatic compounds constitute one of the most populated classes of organic compounds, crucial for pharmaceutical industry, plant protection, perfumery, liquid crystal, OLED, modern functional materials and polymer production, as well as many other chemical applications. The high importance of arenes makes their chemistry enormously rich and diversified. Many different approaches to the synthesis of arenes, which are based on different classes of the reactions, have been developed up to today. Typically, such individual approaches constitute subjects for dedicated books or chapters therein, where the mechanisms, scope and limitations as well as usability of respective approaches are described in detail. Comprehensive titles covering the entire chemistry of arenes, except the chemistry student tutorial texts, have not been published for a long time. Titles covering kinetic studies of arene transformations are even more rare. There was an obvious need for a detailed and comprehensive book, covering the full spectrum of chemistry of benzenoid aromatic compounds, including the new reactions and less obvious approaches, as well as mechanistic discussions and selected theoretical and computational considerations.

The book "Arene Chemistry: Reaction Mechanisms and Methods for Aromatic Compounds" greatly fills this gap. There are ten parts of the book, divided into thirty two chapters, written by the leading, worldwide recognised experts touch on almost all the topics related to the chemistry of arenes. The book covers reactions of aromatic systems formation, dearomatisation reactions, diversified transformation of benzenoid aromatic compounds, introduction and transformation of function groups as well as some aromatic rearrangements.

The classical electrophilic aromatic substitution has been one of the most thoroughly studied organic reaction for about 140 years, and has been covered by many excellent texts. Nevertheless, despite these extensive studies, many important questions remain unanswered. In this book the authors have dedicated five chapters of Part I to that type of arenes reactivity, 
making an untypical selection of the literature to present very important but less discussed topics and examples. The authors also explore the application of $\mathrm{S}_{\mathrm{E}} \mathrm{Ar}$ reaction in total syntheses of natural products, and in several catalytic and enantioselective approaches to arene transformations. In this way, the detailed discussion on the $S_{E} A r$ reactions mechanisms, and quantum chemical theoretical analysis of electrophilic aromatic substitution has been described on a level appropriate for the majority of experienced chemists.

The nucleophilic aromatic substitution is certainly an unappreciated field of aromatic substitution reactions. In student courses, the pallet of $S_{N} A r$ reactions is often limited to the simplest reactions, passing the formation of Meisenheimer's complex intermediate only. In this book, the authors of the corresponding chapters in Part II avoided this uncomprehensive approach; instead they explored different types of $S_{N} A r$ reactions and presented the practical and theoretical aspects of the reaction mechanism including solvent effects as well as effects of activating, leaving, and neighbouring groups. Based on conceptual DFT calculation and experimental kinetic studies, the reactivity of arenes was also explained in terms of quantitative indexes of global and local electrophilicity and nucleophilicity. Several approaches to asymmetric versions of this reaction have also been discussed. In contrast to conventional nucleophilic substitution reaction, the nucleophilic substitution of hydrogen in electron-deficient arenes $\mathrm{S}_{\mathrm{N}} \mathrm{ArH}$ unambiguously follows the fast addition of nucleophiles to nitroarenes (and halonitroarenes) at positions occupied by hydrogen. Thus, in the proper condition, the $\mathrm{S}_{\mathrm{N}} \mathrm{ArH}$ reactions should proceed faster than conventional $S_{N} A r$ ones, giving the hydrogen substitution product (unlike halogen substitution inherent to $S_{N} A r$ ) in excellent yield. Surprisingly, in most textbooks, the nucleophilic substitution of hydrogen is not discussed at all. The corresponding chapters, written by Prof. Mąkosza, are a comprehensive exploration of the utility of the $\mathrm{S}_{\mathrm{N}} \mathrm{ArH}$ reaction, its variations and corresponding mechanisms. The radical substitution reactions, including the radical-nucleophilic aromatic substitution and homolytic aromatic substitution reaction, have proven to be highly efficient for the syntheses of heterocyclic and carbocyclic compounds, especially in intramolecular processes. Sometimes they are considered as an alternative to transition metal-mediated processes. Although the examples shown in the book can only give partial insight into this subject, the authors have clearly illustrated a powerful and synthetic usability of the aromatic radical substitution.
The main types of aryne reactivity along with a discussion of the mechanistic considerations are overviewed in Part III. The usability of these attractive intermediates in different types of the organic transformation has been demonstrated. The readers will obtain sufficient information to get a good general knowledge of the subject which could be also considered as an excellent starting point for further comprehensive exploration.

A very important subject, unfortunately not often comprehensively discussed in general aromatic chemistry courses, are reactions in which the aromaticity of arenes has been destroyed. The discussion on dearomatisation reactions, which are based on oxidative, reductive, or alkylative approaches as well as anionic, radical, and photochemical approaches, has been organised according to the mechanistic considerations with emphasis on the asymmetric processes. This has been presented in Part IV. The authors of the respective chapters make sufficient effort to select the most important examples of the reactions which lead to the important scaffolds of biologically active compounds, drugs, and natural products. The discussion includes both conventional, catalytic, transition metal-mediated, and same enzymatic approaches to the dearomatisation reactions.

Several chapters of Part $V$ are dedicated to the formation of aromatic rings in different type of cyclisation and rearrangement reactions including, among the others, ring-closing metathesis as well as pericyclic reactions and aromatisation leading to the formation of the arene moiety in novo. A wide range of representative applications in the synthesis of aromatic compounds has been demonstrated in the corresponding chapters including several spectacular asymmetric transformations. Several other types of rearrangement reactions are also discussed there.

No modern text on the chemistry of arenes could be complete without the chapter on cross-coupling and $\mathrm{C}-\mathrm{H}$ functionalisation reactions. In this chapter the transition metal-mediated aryl-carbon and aryl-heteroatom bond forming reactions have been carefully explored. Regular, oxidative, and reductive cross-couplings leading through an activation of Ar-Hal, Ar-OX and $\operatorname{Ar}-\mathrm{C}\left(\mathrm{sp}\right.$ and $\left.\mathrm{sp}^{2}\right)$ bonds have been analysed in Parts VI and VII, and many interesting and important examples have been presented. The chelate-assisted and nondirected $\mathrm{C}(\mathrm{Ar})-\mathrm{H}$ transformations are well-represented with multiple examples. The reactions have been arranged according to the hybridisation of the carbon atom (or heteroatom) coupled 
with aryl, transition metal, and cross-coupling reaction mechanism. In line with a deep look into the mechanism, some synthetic applications of the reactions have been discussed. Despite the limited size of the chapters, the data presented in the book allows readers to see a general picture of the state of the art in this field.

An appropriate directing metalation group (DMG) assisted directed metalation of arenes by organolithiums, lithium amides, and other superbases provides an excellent possibility for functionalisation of arenes with unusual and different regioselectivity, than it is expected in the classical $S_{N} A r$ and $S_{E} A r$ reactions. Thus, this method of $\mathrm{C}-\mathrm{H}$ bond activation and subsequent trapping of the nucleophile formed with a wide range of electrophiles is complementary to other better known approaches. Moreover, the directed ortho metalation (DoM), directed remote metalation (DreM), peri metalation of naphthalenes, as well as lateral metalation are often more versatile and more selective than classical methods. A nexus of DoM and cross-coupling reactions leading just in a few reaction steps to the simple and easy formation of polysubstituted aromatics has been also developed. The excellent discussion presented in Part VIII covers the mechanistic issues of DoM, the role of base, solvent, DMG, and electrophile nature as well as the synthetic applications of the methods. The different possibilities of utilisation in the reaction substrates with highly reactive substituents without a need to protect them have also been demonstrated. A supplementary approach to directing ortho metalation are halogen-metal exchange reactions, which are less popular but may constitute an attractive synthetic tool to reverse reactivity of aromatic halides (electrophiles) to metalorganic nucleophiles. All those, as well as some asymmetric reactions, are discussed in several chapters dedicated to reactions of metalation of arenes.

Many well known photochemical reactions of aromatic compounds are frequently applied to organic synthesis. In such reactions the molecules electronically excited by absorption of light undergo the transformations with modified and often inverted reactivity, different from that observed for the compounds in their ground states. Despite these facts, photochemical reactions still need to be better covered in student textbooks and university courses. Part IX contains chapters on photochemical transformations which shed light on that important chemistry with a content score appropriate for wide range of readers.
The Biotransformation of aromatic compounds applied on a preparatively useful scale is still very rare, with their mechanisms significantly less studied than in the case of classical chemical reactions. At the same time, the rapidly rising accessibility of the vast array of enzymes and a continuous attention on environmental concerns facilitates studies on synthetically useful biotransformations of aromatic substrates. Part X, the final section of the book, provides an overview of biocatalytic methods that have been (or might plausibly be) applied to arenes. The transformations presented are categorised by the reaction type, which in combination with the references on both original publication and reviews allow readers to further study this topic on their own, or even to consider biocatalytic processes as a reasonable alternative for classical synthetic processes.

In general, the book is focused only on the most significant and recent results in each topic, since several comprehensive reviews and books containing detailed treatment of these topics have been published in previous years. At the same time, the text provides a view of the complete field of arene chemistry including the reactions of aromatic ring formation, transformation of arenes, and their dearomatisation. The lack of complete deep and exhaustive information in each single topic is rather an advantage than a weakness of the book, as it could be impossible to collect in a single publication, write, or read everything that has been generated up to date on the subject of arene chemistry. The broad covering of topics, made possible by the collaboration of world-renowned experts in different fields of organic chemistry and by a good balance in depth of coverage, positions "Arene Chemistry: Reaction Mechanisms and Methods for Aromatic Compounds" as a deskbook that would be extremely useful for students of advanced courses of organic chemistry, instructors and professors, as well as experienced chemists in both academy and industry, and to those interested in arene chemistry and its application.

Oleg M. Demchuk<0leh.Demchuk@UMCS.Lublin.pl> is assistant professor at the Marie Curie-Skłodowska University in Lublin, Poland. Since 2014, he is the Editor-in-chief of Current Chemistry Letters. As a member of Poland's top 500 innovators program team, he has successfully completed the professional development program in the field of Science Management and Commercialization carried at Stanford University. Prof. Demchuk also has an interest in the philosophy of chemical education. He has been involved in IUPAC since 2007 when he attended his first General Assembly as a Young Observer; he is currently involved in the Organic and Biomolecular Chemistry Division and CHEMRAWN as a titular member. 

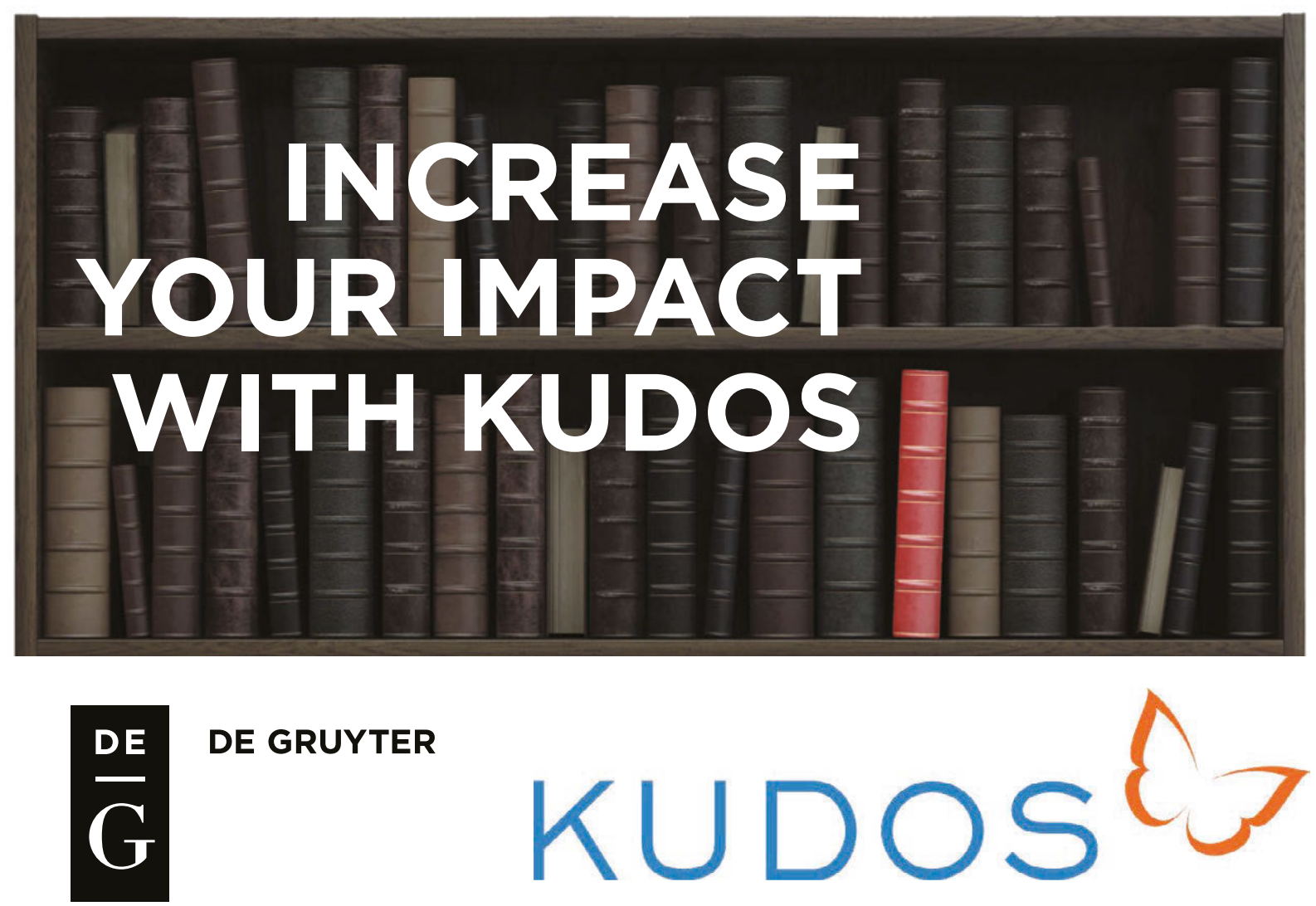

\section{DE GRUYTER}
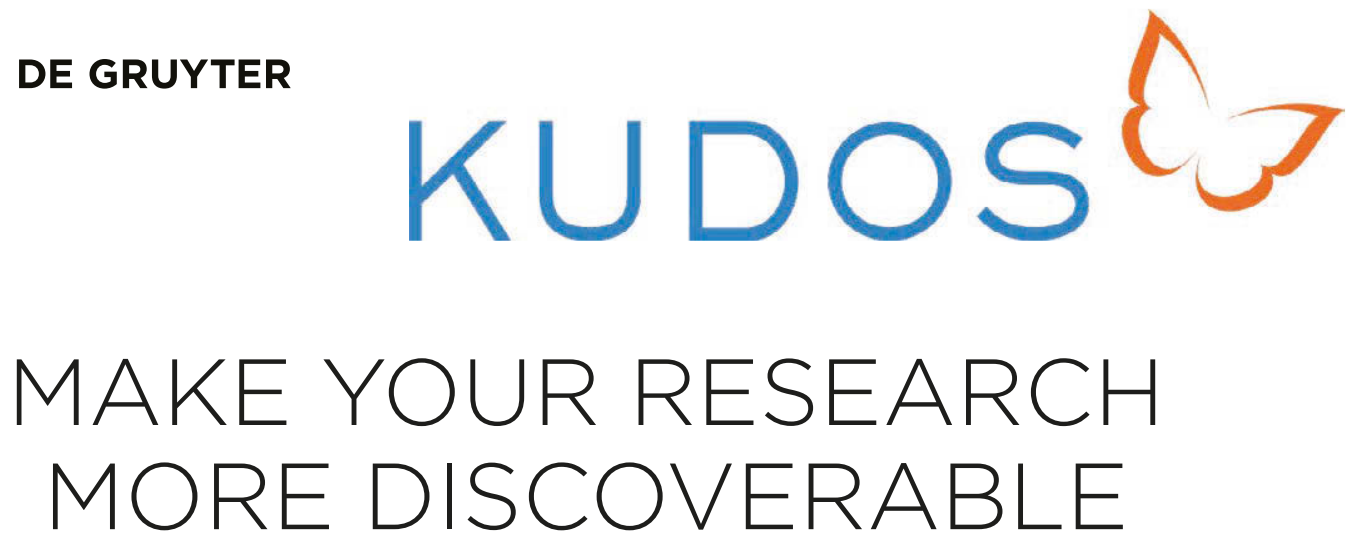

WHAT IS KUDOS?

With more than a million research articles published in the world every year, it is becoming harder for readers to find the latest research quickly. Kudos is an online platform that helps cut through the noise, and makes it easier for researchers to find the latest relevant publications. Created exclusively for authors and researchers, Kudos enables you to promote, explain and enrich your research in order to improve its impact. It also helps you connect with colleagues in related fields.

\section{WHAT ARE THE BENEFITS?}

Kudos is an independent, multi-publisher platform that helps you maximise the exposure of your research by sharing your work with a global network of researchers. It enables you to:

- Increase exposure of your research and improve its impact by reaching the widest possible audience.

- Add context to the published work, including links to supporting information and resources, to increase usage and discoverability

- Create a showcase for your research from all participating publishers

- Integrate comments and contributions from all your co-authors to broaden the influence of your work.

- Drive citations and usage by connecting your work to popular academic and social networks.

- Monitor downloads, shares, views and citations of your work

\section{HOW MUCH DOES IT COST?}

Kudos is free for authors. The costs of running Kudos are covered by publishers including De Gruyter.

\section{Degruyter.com/authors}

